\title{
Correlation Between Intra-Abdominal Free Fluid and Solid Organ Injury in Blunt Abdominal Trauma
}

\author{
Hamidreza Talari ${ }^{1}$; Nushin Moussavi ${ }^{2,}$; Masoumeh Abedzadeh-Kalahroudi ${ }^{2}$; Fatemeh \\ Atoof $^{3}$; Arezoo Abedini $^{1}$ \\ ${ }^{1}$ Department of Radiology, Kashan University of Medical Sciences, Kashan, IR Iran \\ ${ }^{2}$ Trauma Research Center, Kashan University of Medical Sciences, Kashan, IR Iran \\ ${ }^{3}$ Department of Biostatistics and Epidemiology, Tehran University of Medical Sciences, Tehran, IR Iran \\ ${ }^{*}$ Corresponding author: Nushin Moussavi, Department of Surgery, Kashan University of Medical Sciences, Kashan, IR Iran. Tel: +98-3155540026, Fax: +98-3155548900, \\ E-mail:n.moussavi@yahoo.com
}

Received: April 15, 2015; Revised: June 1, 2015; Accepted: June 18, 2015

\begin{abstract}
Background: In previous studies, the diagnostic value of Focused Assessment with Sonography for Trauma (FAST) has been evaluated but few studies have been performed on the relationship between the amount of free intra-abdominal fluid and organ injury in blunt abdominal trauma. To select patients with a higher probability of intra-abdominal injuries, several scoring systems have been proposed based on the results of FAST.

Objectives: The aim of this study was to determine the prognostic value of FAST according to the Huang scoring system and to propose a cut-off point for predicting the presence of intra-abdominal injuries on the Computed Tomography(CT) scan. The correlation between age and Glasgow Coma Scale(GCS) and the presence of intra-abdominal injuries on the CT scan was also assessed.

Patients and Methods: This study was performed on 200 patients with severe blunt abdominal trauma who had stable vital signs. For all patients, FAST-ultrasound was performed by a radiologist and the free fluid score in the abdomen was calculated according to the Huang score. Immediately, an intravenous contrast-enhanced abdominal CT scan was performed in all patients and abdominal solid organ injuries were assessed. Results were analyzed using Kruskal-Wallis test, Mann-Whitney test and ROC curves. The correlation between age and GCS and the presence of intra-abdominal injuries on CT-scan was also evaluated.

Results: The mean age of the patients was $29.6 \pm 18.3$ years and FAST was positive in $67 \%$ of the subjects. A significant correlation was seen between the FAST score and the presence of organ injury on CT scan $(\mathrm{P}<0.001)$. Considering the cut-off point of 3 for the free fluid score (with a range of 0-8), sensitivity, specificity, positive predictive value and negative predictive value were calculated to be $0.83,0.98,0.93$, and 0.95, respectively. Age and GCS showed no significant correlation with intra-abdominal injuries.

Conclusions: It seems that FAST examination for intra-abdominal fluid in blunt trauma patients can predict intra-abdominal injuries with very high sensitivity and specificity. Using the scoring system can more accurately determine the probability of the presence of abdominal injuries with a cut-off point of three.
\end{abstract}

Keywords: Blunt Trauma; Ultrasound; FAST; Free Fluid

\section{Background}

Trauma is the fourth leading cause of death among people in developing countries like Iran; it is the second leading cause of death in young people $(1,2)$. Despite the improvement in medical care, trauma is still a leading cause of death all over the world. Abdominal injuries are a common cause for death and are seen in $20 \%$ of trauma patients $(3,4)$. Imaging of the abdomen, pelvis and spine in trauma patients is very important as clinical examination alone is not sensitive enough to detect life-threatening injuries. Early detection of intraabdominal hemorrhage can save lives; therefore, imaging of the abdomen using ultrasound and CT scan is usually used in blunt trauma patients to detect occult injuries and hemorrhage before becoming life-threat- ening. Focused Assessment with Sonography for Trauma (FAST) was first described by Rozycki et al. in 1990 as an accurate, noninvasive and cost-effective diagnostic tool for severely injured trauma patients in the emergency department (5). Also, FAST can be used bedside and is quickly available in emergency situations. The intra-abdominal and cardiac regions can be evaluated in 5 minutes and in comparison to X-ray and CT scan, it is not associated with radiation exposure. Therefore, FAST is nowadays the first step in evaluation of intraabdominal hemorrhage in trauma patients. With the invention of a rapid sequence helical CT scan, a new era begun for detecting intra-abdominal injuries. Computed tomography scan is more sensitive than ultrasound

Copyright (C) 2015, Kashan University of Medical Sciences. This is an open-access article distributed under the terms of the Creative Commons Attribution-NonCommercial 4.0 International License (http://creativecommons.org/licenses/by-nc/4.0/) which permits copy and redistribute the material just in noncommercial usages, provided the original work is properly cited. 
and can show more details and occult injuries $(6,7)$. Although often used in equipped trauma centers, the radiation exposure and higher costs limit the routine use of this sensitive diagnostic tool (8-10).

\section{Objectives}

To select patients with a higher probability of intraabdominal injuries requiring laparotomy, several scoring systems have been proposed based on the results of FAST and other clinical data. The aim of this study was to determine the prognostic value of FAST according to the Huang scoring system as well as to propose a cut-off point for predicting the presence of intra-abdominal injuries.

\section{Patients and Methods}

This cross-sectional study was performed on 200 hemodynamically stable patients with severe blunt trauma referred to the emergency department of Shahid Beheshti hospital in Kashan, Iran. Severe trauma was assumed if the mechanism of trauma was high energy (falls more than 3 meters, motor vehicle accident with a speed more than $50 \mathrm{Km} / \mathrm{h}$, crush injuries, rollover and pedestrian accidents) or if severe injuries such as vertebral or pelvic fractures were detected. Hemodynamically unstable patients (systolic blood pressure $<90 \mathrm{~mm} \mathrm{Hg}$ ), penetrating trauma patients, pregnant women and those with underlying diseases associated with intra-abdominal fluid (cirrhosis, congestive heart failure) were excluded from the study. The study population was calculated to be 197 patients according to ultrasound sensitivity of $85 \%$ for detecting free intra-abdominal fluid, accuracy of $95 \%$ and d of 0.05. All patients were evaluated by FAST in the supine position with the Medison ultrasound (V20) curvilinear 3.5 - 5 $\mu \mathrm{HZ}$ transducer. For measurement of the intra-abdominal fluid the Huang classification was used. One score was given for each intra-abdominal region (Douglas pouch, hepatorenal recess, perisplenic and paracoloc gutters) if free intra-abdominal fluid was present. If free fluid more than $2 \mathrm{~mm}$ was seen in the hepatorenal recess and Douglas pouch or floating bowel loops were seen, two points were given. So, a maximum score of 8 could be reached (11), Table 1. After performing ultrasound, abdominopelvic CT scan (Toshiba-Astion) was performed for all patients from the diaphragm dome to pubis symphysis with intravenous contrast (Visipaque $270 \mathrm{mg}$ vial, $1 \mathrm{cc} / \mathrm{Kg}$ ). The $\mathrm{CT}$ scan was reported by a second radiologist who was blinded to the results of the ultrasound. Results were analyzed using logistic regression, Kruskal-Wallis and Mann-Whitney tests. For obtaining the cut-off point, ROC curves were used. A P value of less than 0.05 was considered to be statistically significant.

\section{Results}

Two-hundred blunt abdominal trauma patients were included in the study. The mean age of the patients was $29.6 \pm 18.3$ years (median $=18.3$ ) and 133 patients $(66.5 \%)$ were male. Mean GCS at arrival in the emergency room was $12.9 \pm 3.4$ (median $=15)$. The trauma mechanism was motor vehicle accident in $71 \%$ of the cases. Also, FAST was positive in $67 \%$ of subjects (Table 2 ). The results of the intra-abdominal fluid score of the patients is shown in Figure 1. Comparing the mean scores from FAST and the CT scan results showed that there was a significant correlation between the presence of organ injury and the FAST scores $(\mathrm{P}<0.001)$. To determine an appropriate cutoff point to predict the probability of intra-abdominal injury, the ROC curve was used (Figure 2). Considering the cut-off point of 3 for the free fluid score, sensitivity, specificity, positive and negative predictive value were calculated to be $0.83,0.98,0.93$, and 0.95 , respectively (Table 3). Age and GCS score showed no significant correlation with the presence of intra-abdominal injury on CT scan with p-values of 0.27 and 0.68 , respectively.

Table 1. The Huang Classification for Intra-Abdominal Free Fluid

\begin{tabular}{lcc}
\hline Anatomic Area & Amount of Fluid & Points \\
\hline Morrison's pouch & $\leq 2 \mathrm{~mm}$ & 1 \\
\hline Douglas pouch & $>2 \mathrm{~mm}$ & 2 \\
\hline Parasplenic space & $\leq 2 \mathrm{~mm}$ & 1 \\
\hline Paracolic gutter & $>2 \mathrm{~mm}$ & 2 \\
\hline Floating intestinal loops & Any & 1 \\
\hline
\end{tabular}

Table 2. Baseline Characteristics of the Study Population ${ }^{\text {a }}$

\begin{tabular}{lc}
\hline Characteristics & Values \\
\hline Age, $\mathbf{y}$ & $29.6 \pm 18.3$ \\
\hline GCS $^{\mathbf{b}}$ & $12.9 \pm 3.4$ \\
\hline Gender, male & 66.5 \\
\hline Trauma mechanism & $142(71)$ \\
\hline \multicolumn{2}{l}{ Motor vehicle accident } \\
\hline \multicolumn{2}{l}{ Other (falls, hits) } \\
\hline $\begin{array}{l}\text { a Values are presented as mean } \pm \text { SD, or \% or No. (\%). } \\
\text { b GCS, Glasgow coma scale. }\end{array}$
\end{tabular}


Talari Het al.

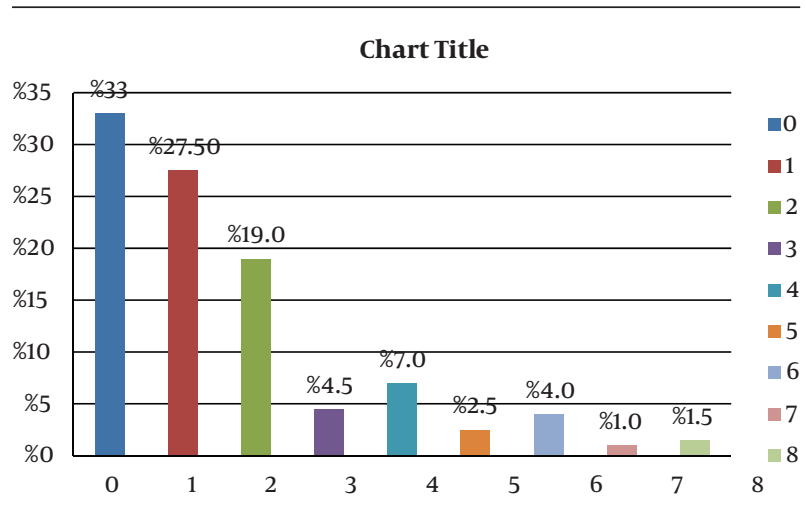

Figure 1. Distribution of Huang Intra-Abdominal Fluid Score in the Patient Population

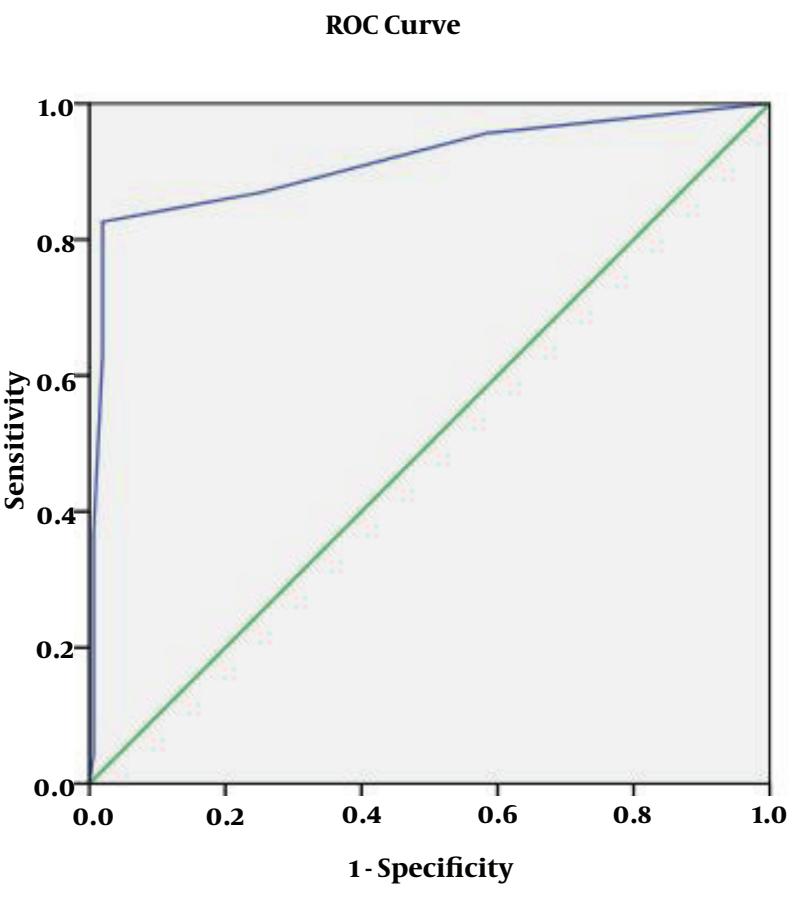

Figure 2. ROC Curve Showing Sensitivity and Specificity of the Huang Scoring System in Predicting an Intra-Abdominal Injury

Table 3. Sensitivity and Specificity of Different Points of the Huang Classification; Point Three Was Considered as the Most Suitable Cut-Off Point

\begin{tabular}{lll}
\hline Huang Score & Sensitivity & Specificity \\
\hline $\mathbf{2}$ & 0.87 & 0.75 \\
$\mathbf{3}$ & 0.93 & 0.98 \\
$\mathbf{4}$ & 0.63 & 0.98 \\
\hline
\end{tabular}

\section{Discussion}

The sensitivity and specificity of FAST in our study were $83 \%$ and $98 \%$, respectively with a positive predictive value of $93 \%$ and a negative predictive value of $95 \%$. In the study performed by Lingawi and Buckley (12) on 1090 patients, the sensitivity, specificity, and positive and negative predictive values for FAST were reported 94\%, 98\%, 78\% and $100 \%$, respectively. Similar results were reported by Foo et al. (13) showing sensitivity and specificity of $86 \%$ and $92 \%$, respectively and positive and negative predictive values of $89 \%$. Also, Mckenney et al.(14) showed a sensitivity of $83 \%$ and specificity of $87 \%$ for the intra-abdominal fluid score to predict solid organ injuries. Similar results are reported in several studies (15-17). On the other hand, there are also some studies depicting a lower sensitivity for FAST. Natarajan et al. (18) could only reveal a sensitivity of $41 \%$ in a retrospective study of 2980 trauma patients; the specificity was reported to be $99 \%$. Moreover, other studies showed low sensitivities of 54\% and 50\% with specificities of $90 \%$ and $97.3 \%(19,20)$. In one study performed in children, a sensitivity of $55 \%$ and a specificity of $83 \%$ were observed. Ten out of 107 patients had a solid organ injury despite a normal FAST examination. The authors concluded that FAST is not sensitive enough in children to predict intra-abdominal injuries (21). The results of our study revealed very good sensitivity, specificity and positive and negative predictive values. Differences in sensitivity seen in the several studies may be attributable to different patient populations and the skill and precision of the sonographist. In children additional factors may influence the results, such as poorer cooperation or anatomical differences.

To predict the probability of emergency abdominal surgery in trauma patients, several scoring systems exist. Huang et al. (11) used a scoring system based on the presence of fluid in different areas and its amount as previously mentioned. In their study, $96 \%$ of patients with a score of three or more required laparotomy (11). A similar score for the amount of the intra-abdominal fluid was proposed by McKenney et al. (14) in 2001, showing a sensitivity of $83 \%$ for the "hemoperitoneum score" in determining the need for a therapeutic laparotomy. In their study, ultrasound revealed an obvious better sensitivity in contrast to systolic blood pressure or base deficit (sensitivity values of $28 \%$ and $49 \%$, respectively) to predict the need for laparotomy. Eighty-seven percent of trauma patients with a score of three or more underwent laparotomy, but only $15 \%$ of those had a score of less than three (14). Also, Moylan et al. (22) showed that a positive FAST can predict the probability of emergency surgery very well; $37 \%$ of hemodynamically stable trauma patients with a positive FAST examination underwent laparotomy in contrast to only $0.5 \%$ in those with a normal ultrasound. This author did not use any scoring system. In our study, similar to the above-mentioned studies, the amount of free fluid correlated with the presence of intra-abdominal injuries 
and a cut-off point of three was obtained, depicting that a score of 3 or more is associated with a high probability of intra-abdominal injuries.

Other clinical data are also used in other scoring systems to predict the probability of organ injury or need for laparotomy. McKenney et al. (14) compared the sensitivity of ultrasound to predict intra-abdominal injuries with systolic blood pressure and base deficit. The sensitivity of ultrasound in determining the need for operative intervention was $83 \%$, remarkably higher than blood pressure (28\%) and base deficit (49\%) (14). Manka et al. (23) used a scoring system based on the amount of intra-abdominal fluid, blood pressure and pulse rate, revealing a sensitivity of $98.2 \%$ and a specificity of 70\%. Musiitwa et al. (24) used the SSORTT score (sonographic scoring for operating room triage in trauma) based on FAST, systolic blood pressure and pulse rate and showed that patients with a SSORTT score of 2 and above had a high likelihood of requiring a therapeutic laparotomy. We could not see any correlation between level of consciousness (according to GCS) and the presence of intra-abdominal injuries. Considering the results of the mentioned studies ultrasound seems to be much more sensitive in predicting intra-abdominal injuries than any other clinical or para-clinical parameters.

In conclusion, FAST examination for intra-abdominal fluid in blunt trauma patients can predict intra-abdominal injuries with a very high sensitivity and specificity. Using the Huang scoring system can more accurately determine the probability of the presence of abdominal injuries with a cut-off point of three. Age and GCS have not been predictive of intra-abdominal injuries, but further studies in the future should focus on combining other clinical or para-clinical factors with FAST to design a more sensitive scoring system to predict intra-abdominal injuries and need for laparotomy.

\section{Acknowledgements}

We would like to thank Kashan University of Medical Sciences and Trauma Research Center for their support of this study.

\section{Authors' Contributions}

All authors contributed to the work and meet the criteria for authorship.

\section{Funding/Support}

This work was supported by the trauma research center and the deputy of research of Kashan University of Medical Sciences (grant number: 9130).

\section{References}

1. Ahmadi Amoli H, Zafarghandi MR, Tavakoli H, Davoodi M, Khashayar P. Thoracic trauma: severity of injury in 342 patients. Tehran Univ Med Sci. 2009;66(11):831-4.

2. Hemmati H, Kazemnezhad-Leili E, Mohtasham-Amiri Z, Darzi AA, Davoudi-Kiakalayeh A, Dehnadi-Moghaddam A, et al. Evaluation of chest and abdominal injuries in trauma patients hospitalized in the surgery ward of poursina teaching hospital, guilan, iran. Arch Trauma Res. 2013;1(4):161-5.

3. Khan IA, Ghaffar S, Asif S, Zia ur R, Asad S, Ahmed E, et al. Management of thoracic trauma: experience at Ayub Teaching Hospital, Abbottabad. J Ayub Med Coll Abbottabad. 2009;21(1):152-4.

4. Mefire AC, Pagbe JJ, Fokou M, Nguimbous JF, Guifo ML, Bahebeck $\mathrm{J}$. Analysis of epidemiology, lesions, treatment and outcome of 354 consecutive cases of blunt and penetrating trauma to the chest in an African setting. S Afr J Surg. 2010;48(3):90-3.

5. Helling TS, Wilson J, Augustosky K. The utility of focused abdominal ultrasound in blunt abdominal trauma: a reappraisal. Am J Surg. 2007;194(6):728-32.

6. Victorino GP, Chong TJ, Pal JD. Trauma in the elderly patient. Arch Surg. 2003;138(10):1093-8.

7. Fang JF, Wong YC, Lin BC, Hsu YP, Chen MF. Usefulness of multidetector computed tomography for the initial assessment of blunt abdominal trauma patients. World J Surg. 2006;30(2):176-82.

8. Brenner DJ, Hall EJ. Computed tomography--an increasing source of radiation exposure. $N$ Engl J Med. 2007;357(22):2277-84.

9. Ott M, McAlister J, VanderKolk WE, Goldsmith A, Mattice C, Davis AT. Radiation exposure in trauma patients. J Trauma. 2006;61(3):607-9.

10. Tien HC, Tremblay LN, Rizoli SB, Gelberg J, Spencer F, Caldwell C, et al. Radiation exposure from diagnostic imaging in severely injured trauma patients. JTrauma. 2007;62(1):151-6.

11. Huang MS, Liu M, Wu JK, Shih HC, Ko TJ, Lee CH. Ultrasonography for the evaluation of hemoperitoneum during resuscitation: a simple scoring system. J Trauma. 1994;36(2):173-7.

12. Lingawi SS, Buckley AR. Focused abdominal US in patients with trauma. Radiology. 2000;217(2):426-9.

13. Foo E, Su JW, Menon D, Tan D, Chan ST. A prospective evaluation of surgeon performed sonography as a screening test in blunt abdominal trauma. Ann Acad Med Singapore. 2001;30(1):11-4.

14. McKenney KL, McKenney MG, Cohn SM, Compton R, Nunez DB, Dolich $\mathrm{M}$, et al. Hemoperitoneum score helps determine need for therapeutic laparotomy. J Trauma. 2001;50(4):650-4.

15. Tsui CL, Fung HT, Chung KL, Kam CW. Focused abdominal sonography for trauma in the emergency department for blunt abdominal trauma. Int J Emerg Med. 200 8;1(3):183-7.

16. Goletti O, Ghiselli G, Lippolis PV, Chiarugi M, Braccini G, Macaluso $\mathrm{C}$, et al. The role of ultrasonography in blunt abdominal trauma: results in 250 consecutive cases. J Trauma. 1994;36(2):178-81.

17. Hamed-Barghi G, Mohammadi A, Safaeian A. Diagnostic value of sonogeraphy in blunt abdominal trauma. Ardabil Uni Med Sci. 2005;217(2):426-9.

18. Natarajan B, Gupta PK, Cemaj S, Sorensen M, Hatzoudis GI, Forse RA. FAST scan: is it worth doing in hemodynamically stable blunt trauma patients? Surgery. 2010;148(4):695-700.

19. Rohani Z, Naroee B, Taymori MA, Gasemi Rad M, Mohebi F, Shaykhzadeh. [Comparison of Sensitivity and Specificity of Sonography with CT Scan in the Diagnosis of Abdominal Free Fluid in Blunt Abdominal Trauma in Khatamol-Anbia Hospital, Zahedan]. Iran J Surg. 2009;17(3):32-8.

20. Cheung KS, Wong HT, Leung LP, Tsang TC, Leung GK. Diagnostic accuracy of Focused Abdominal Sonography for Trauma in blunt abdominal trauma patients in a trauma centre of Hong Kong. Chin JTraumatol. 2012;15(5):273-8.

21. Coley BD, Mutabagani KH, Martin LC, Zumberge N, Cooney DR, Caniano DA, et al. Focused abdominal sonography for trauma (FAST) in children with blunt abdominal trauma. J Trauma. 2000;48(5):902-6.

22. Moylan M, Newgard CD, Ma OJ, Sabbaj A, Rogers T, Douglass R. Association between a positive ED FAST examination and therapeutic laparotomy in normotensive blunt trauma patients. J Emerg Med. 2007;33(3):265-71.

23. Manka M, Moscati R, Raghavendran K, Priya A. Sonographic scoring for operating room triage in trauma. West J Emerg Med. 2010;11(2):138-43.

24. Musiitwa PC, Galukande M, Bugeza S, Wanzira H, Wangoda R. Emergency Ultrasound Predicting the Need for Therapeutic Laparotomy among Blunt Abdominal Trauma Patients in a SubSaharan African Hospital. Emerg Med Int. 2014;2014:793437. 